\begin{tabular}{|c|l|}
\hline Title & Expression of Foreign Genes in Lamprey Embry os: An A pproach to Study Evolutionary Changes in Gene Regulation \\
\hline Author(s) & Kusakabe, Rie; Tochinai, Shin; Kuratani, Shigeru \\
\hline Citation & $\begin{array}{l}\text { Journal of Experimental Zoology Part B: Molecular and Developmental Evolution, 296B(1), 87-97 } \\
\text { https:/doi.org/L0.1002/3z.b.11 }\end{array}$ \\
\hline Issue Date & $2003-03$ 20 \\
\hline Doc URL & http://hdl.handle.net/2115/540 \\
\hline Rights & Copyright (c) 2003Wiley-Liss, Inc. \\
\hline Type & article (author version) \\
\hline File Information & JEZ296B.pdf \\
\hline
\end{tabular}

Instructions for use 


\title{
Expression of Foreign Genes in Lamprey Embryos: An Approach to Study Evolutionary Changes in Gene Regulation
}

\author{
RIE KUSAKABE1,2, SHIN TOCHINAI2, AND SHIGERU KURATANI1 \\ 1Laboratory for Evolutionary Morphology, Center for Developmental Biology, RIKEN, Japan \\ ${ }_{2}$ Department of Biological Sciences, Graduate School of Science, Hokkaido University, Japan
}

\begin{abstract}
Evolution in development can be viewed as a sequence of changes in gene regulation. To investigate the cross-species compatibility of 5' upstream regulatory regions, we introduced exogenous gene constructs derived from a gnathostome genome into fertilized eggs of the Japanese lamprey, Lampetra japonica, a sister group of the gnathostomes. Eggs were injected with gene constructs in which a sequence encoding the green fluorescent protein (GFP) had been located downstream of either a virus promoter or 5' regulatory regions of medaka actin genes. Reporter gene expression was recorded for more than a month starting two days after injection. Although the expression patterns were highly mosaic and differed among individuals, GFP was expressed predominantly in the striated muscles of lamprey embryos when driven by the 5' upstream regions of the medaka muscle actin genes. This implies that a pan-vertebrate muscle-specific gene regulatory mechanism may have evolved before the agnathan/gnathostome divergence. This gene-transfer technique potentially facilitates the visualization of cells in various differentiating tissues throughout development. The introduction of developmental genes of the lamprey or other animals into lamprey embryos is another potentially important application, one that could provide us with information on the evolutionary changes in functions of genes or gene cascades. J. Exp. Zool. (Mol.Dev. Evol.) 296B:87-97, 2003.
\end{abstract}

\section{INTRODUCTION}

Exogenous gene transfer has been a commonly used strategy for the analysis of gene function in some animals that are used as "developmental model systems," such as the mouse (Mu"ller, '99), zebrafish (Higashijima et al., '97; Krovel and Olsen, 2002), C. elegans (Mello and Fire, '95), and Drosophila (Rong et al., 2002). Although recent advances include new, sophisticated technology such as electroporation (Swartz et al., 2001; Di Gregorio and Levine, 2002) and sperm nuclear transfer (Kroll and Amaya, '96; Jesuthasan and Subburaju, 2002), the most conventional method with which to generate transgenic animals is still the simple microinjection of a gene into the cytoplasm or nucleus of a fertilized egg. Injected individuals are raised to maturity and crossed to produce stable transgenic lines in which the foreign genes are expected to be transmitted in the germ line. This type of strategy is complicated and time-consuming, and difficult to apply to wild animals, which occupy important positions in animal phylogenies.

Previously, the expression of reporter genes injected into embryos was analyzed directly to determine tissue-specific regulatory regions in sea urchins (Davidson, '99), ascidians (Kusakabe, '97; Harafuji et al., 2002), and the teleost medaka, Oryzias latipes (Kusakabe et al., '99; Kusakabe and Suzuki, 2001). In these analyses, reporter constructs were generated by fusing upstream regulatory regions of tissue-specific genes to the coding regions of reporter genes such as lacZ or GFP. Expression is supposedly transient, but it precisely reflects the expression pattern of the endogenous genes. By microinjecting various constructs carrying deletions or mutations in regulatory sequences, the components of regulatory regions can be identified in relatively short times, compared with the use of genetically established transgenic lines.

The lamprey, an agnathan (jawless) vertebrate species, is considered to be a sister group of the gnathostomes (jawed vertebrates; Janvier, '96). In previous molecular phylogenetic studies, lamprey 
genes have often been positioned on phylogenetic trees as the sister groups of all the gnathostome genes (Mallatt and Sullivan, '98; Kuraku et al., '99). Recent analysis of lamprey development at the molecular level seems to have clarified the evolutionary sequences of the changes in regulation of some developmentally important regulatory genes (Ogasawara et al., 2000; Murakami et al., 2001; Myojin et al., 2001; Shigetani et al., 2002; Cohn, 2002; reviewed by Kuratani et al., 2001, 2002). However, it has been difficult to test the functions of gene products or gene regulatory regions in live lamprey embryos. In this paper, we report the introduction of exogenous gene constructs into fertilized lamprey eggs by microinjection. The validity of this strategy was demonstrated in cross-specific gene transfer between medaka and lamprey using the upstream regions of muscle actin genes.

As the first practical application of this transgenic method, we also demonstrate in this paper a detailed observation of differentiating skeletal musculature that possesses both pan-vertebrate and lamprey-specific characteristics. The vertebrate skeletal musculature can be roughly classified into two major categories: axial trunk muscles derived from postotic somites, and head muscles, which originate from the head mesoderm. In the gnathostomes, the former can further be categorized as epaxial and hypaxial muscles, whereas the latter include pharyngeal muscles and extrinsic ocular muscles.

Visualization of these members of the lamprey musculature has been performed using acetylcholinesterase (AchE) activity and the monoclonal antibody $\mathrm{CH}-1$ raised against tropomyosin (Kuratani et al., '97, '99). These markers appear in the head muscle much later than in the paraxial myotomes. Here, based on the muscle-specific expression pattern of introduced GFP gene, we contribute new insights into the development of the lamprey musculature visualized in living embryos.

\section{MATERIALS AND METHODS}

\section{Obtaining lamprey embryos}

Ripe adults of the Japanese lamprey Lampetra japonica were purchased from the Ebetsu Fishermen's Association (Ebetsu, Hokkaido, Japan), and maintained at 141C. Spawning was induced, and fry were reared to the appropriate developmental stages at $161 \mathrm{C}$ in fresh water or in $10 \%$ Steinberg's solution (Steinberg, '57). Developmental stages were defined according to the description of Tahara ('88) for $L$. reissneri, a species closely related to L. japonica.

\section{Microinjection of foreign genes}

The pEGFP-N1 construct (Clontech) consists of the cytomegalovirus (CMV) promoter and enhanced GFP coding region. The structures of the fusion genes OlMA1-GFP and OlMA2-GFP, which consist of the 5' upstream regions of the medaka muscle actin genes and the GFP coding region, were described by Kusakabe et al. ('99; Fig. 1A). OlMA1-GFP corresponds to MA1(- 949) and OlMA2-GFP corresponds to MA2(-1570) (Kusakabe et al., '99).

Micropipettes were made from siliconized and sterilized 1_90-mm fiber-filled glass capillary tubes (GD-1, Narishige) using a horizontal puller. Circular plasmid DNA was dissolved in phosphatebuffered saline (PBS) containing $0.1 \%$ phenol red at concentration on $50 \mathrm{ng} / \mathrm{ml}$. Under a dissecting microscope, fertilized eggs were stabilized by suction using a glass holding pipette and injected with DNA solution through the chorion. The injected eggs were washed and incubated in 1_Steinberg's solution (Steinberg, '57) at 161C. GFP signal was detected under a stereoscopic fluorescence microscope. Most of the embryos did not hatch spontaneously, and the chorion had to be removed manually.

\section{RESULTS}

\section{Introduction of exogenous gene constructs into lamprey embryos}

In L. japonica, it usually takes about $6 \mathrm{~h}$ from fertilization to the first cleavage. During this period, fertilized eggs were injected with DNA constructs containing various promoter sequences and the GFP 
coding sequence. About half the injected embryos survived for more than two days. Most of the embryos displayed normal morphology, but some were severely deformed.

In the initial experiment, pEGFP-N1, in which the CMV promoter is attached to the coding region of modified GFP, was injected. A total of 207 embryos were injected; 185 survived for longer than three days. In 80 embryos among them, strong GFP expression was observed from stage 10 (day 2) onwards (Fig. 2A-E). The expression patterns varied from embryo to embryo. Up to the neurula stage, regional GFP expression was detected in random and asymmetrical patterns (Fig. 2A), implying that these cells derived from a small number of blastomeres that had incorporated the exogenous genes. In the pharyngula, reporter gene expression was seen in multiple types of cells, predominantly in yolk cells and epidermal cells (Fig. 2B). As development proceeded, signal was often seen in separate cell populations within an embryo (Fig. 2C). Although pEGFP-N1 was often activated more strongly in epidermal and yolk cells than in other cell types, activation of the CMV promoter did not exhibit any strict tissue preference. There were some examples of organ-specific expression of pEGFPN1. These included an embryo with specific GFP expression in the pronephric duct (Fig. 2D) and another expressing GFP in the trunk skeletal musculature (Fig. 2E).

\section{Expression of medaka muscle actin-GFP constructs in lamprey embryos}

The above experiments demonstrated that exogenous DNA constructs can be injected into fertilized lamprey eggs and stably expressed throughout development. To examine whether promoters of tissue-specific genes originating from other animal species are functional in the lamprey embryo, we injected gene constructs carrying an upstream region of medaka muscle actin genes connected to the GFP coding sequence (Fig.1A) into fertilized lamprey eggs. We used two different medaka muscle actin genes, the expression patterns and functions of the 5' regulatory regions of which have been studied previously (Kusakabe et al., '99). The skeletal muscle actin gene, OlMA1, is expressed specifically in somitic skeletal muscle in medaka embryos. It has been shown in this animal that the genomic region from $=949$ to the start codon of OlMA1, which includes the first intron, is sufficient for skeletal muscle-specific expression of a GFP reporter (Fig. 1B). Another medaka muscle actin gene, OlMA2, is the cardiac actin gene, which is expressed in both somitic and cardiac muscles. The upstream region from -1570 to the start codon of OlMA2, which includes the first intron, is sufficient for specific expression in both skeletal and cardiac muscles (Fig. 1C). In the present study, we designated these regions connected to the GFP coding sequence as OlMA1- GFP and OlMA2-GFP.

The OlMA1-GFP plasmid was injected into fertilized lamprey eggs before the first cleavage. Five days after injection, weak GFP expression started in broad areas of the embryos including epidermal cells. Ten GFP-positive embryos out of 58 total injected embryos were examined extensively. As soon as the somites formed at stage 22, GFP expression was detected in several parts of the somites in 5 embryos (Fig. 2F). Expression was not seen in the heart of any of these embryos, consistent with the results of experiments using the same construct in medaka (Kusakabe et al., '99). The other five embryos died before the formation of somites. As the development proceeded, GFP expression in somites became stronger and more distinctive than that in other parts of the embryos. GFP-positive areas often consisted of adjacent cells from different somites that appeared to have been derived from a part of the pre-segmented mesoderm. The expression patterns in somitic muscle were highly mosaic, and differed between the left and right sides of the embryos (Fig. 2G and H). Such a GFP expression pattern was maintained even after the embryos grew into ammocoete larvae.

Similarly, OlMA2-GFP was injected into 69 fertilized lamprey eggs and 10 GFP-positive embryos were served for detailed observation. GFP was initially expressed in the epidermal cells, and then in forming somites of five embryos (Fig. 3B). As soon as the heart formed (stage 25), strong GFP expression was visible in the pulsating myocardium (Fig. 4B). GFP expression specific to the skeletal and cardiac muscle continued into the ammocoete larval stage, again consistent with the expression pattern of the same construct in medaka embryos (Kusakabe et al., '99). The above results show that 
transcriptional regulatory mechanisms of skeletal and cardiac muscle-specific actin genes are conserved between medaka and lamprey.

In these experiments, both OlMA1- and OlMA2- GFP were also ectopically expressed in epidermal cells, especially at the initial stage of the reporter gene expression. This ectopic expression mirrors that of the same transgenes in medaka (Kusakabe et al., '99). Moreover, in medaka, the ectopic epidermal GFP signal was constantly observed, even in embryos injected with shorter constructs that exhibited no GFP signal in muscle cells (Kusakabe et al., '99). Thus the embryonic epidermal cells might serve as an environment in which the 5' upstream regions carrying minimal transcriptional regulatory elements could be activated.

\section{Development of lamprey head muscle as visualized by OIMA2-GFP}

Two of the OlMA2-GFP-injected embryos, \#1 and \#7, survived for longer than one month with marked expression of GFP. In addition to the initial expression seen similarly in somites and heart muscle, embryo \#1 and \#7 displayed signals in different cell populations within head skeletal muscle derivatives.

Embryo \#1 starts to express strong signals in myotomes as soon as they are formed (Fig. 3B and C). As the development proceeds, strong patchy signals appeared to cover the dorsal part of the head (Fig. 3G), most of which we speculate as somitic derivatives, since they characteristically extend rostrally to cover the lateral aspect of the embryonic head (see discussion). In addition to the signal in the epaxial somitic muscle, embryo \#1 expressed specific signals in muscle fibers in branchial arches 4 to 7 (Fig. 3F and G). Within the bundle of muscle fibers in branchial arch 4, several distinct muscle fibers were identified with strong GFP signal (Fig. 3F and G). Two sets of muscle fibers extended dorsoventrally; one was the constrictor branchial muscle located lateral to the branchiopore, and the other was the adductor branchial muscle, which is located medial to the former. Between the constrictor and adductor muscles, the branchiopore is surrounded by the anterior and posterior external branchial sphincter muscle. In our observations, the sphincter muscle first appeared as a complete ring, within which the anterior and posterior parts of this muscle could not be distinguished. These branchial muscle cells seemed to have been derived from the preotic head mesoderm (Kuratani et al., '99).

On the lateral aspect of the pharynx, segmented hypobranchial muscle extends rostrally. Precursors of this muscle appear to originate from postotic epibranchial somites, which migrate first caudally, ventrocaudally, and ventrally, and then rostrally along the entire length of the pharynx beneath the gill pores (Kuratani et al., '97, '99). After reaching the lateral aspect of the pharynx, this muscle divides into segments (reviewed by Kuratani et al., '97). The GFP signal in this muscle of embryo \#1 appeared much later than signals in other branchial musculatures.

When embryo \#1 was fully grown into an ammocoete larva (Fig. 3D-G), these GFP-positive muscles exhibited concerted movement in expanding and compressing the gill chamber, producing a flow of water from the velum through the gill chamber. These visceral muscles in the ammocoete are thought to disappear to be replaced by adult muscles during metamorphosis (Hardisty and Rovainen, '72). Embryo \#7 had strong GFP signal in somite rows on both sides of the body (Fig. 4A and B). More somite blocks produced signal on the right side than on the left. The rostral part of the embryo was somewhat deformed morphologically; an abnormal protrusion at the rostral end, with no mouth or lips and no eye (Fig. 4D). At stage 25, the most rostral GFP-positive somite on the right side corresponded to the second somite, judging from its dorsally split and anteriorly extended morphology (Kuratani et al., '99; Fig. 4C). The dorsal half gave rise to the supraoptic muscle, and the ventral half to the infraoptic muscle, both of which expressed strong GFP signal in the ammocoete larval stage (Fig. 4F). The supraoptic muscle seemed to consist of an anterior part extending into the rostral tip of the animal, and a posterior part dorsally overlapping the anterior part (Fig. 4G). The third somite did not express GFP until stage 25 (Fig. 4C, black arrowhead), but it developed a small number of GFP-positive fibers at later stages (Fig. 4F, black arrowhead). 


\section{DISCUSSION}

\section{Exogenous gene transfer by microinjection applied to lamprey embryos}

Because our understanding of developmental mechanisms has been greatly accelerated by the application of transgenic technology in mice, more emphasis has been placed on the effective introduction of exogenous genes into the germ line in many animal species used in laboratories. In studies of marine invertebrates, however, exogenous genes can be expressed quite stably according to the spatiotemporal specificity of their promoters, when simply injected into fertilized eggs. Although it is inevitable that this expression will be mosaic, this method potentially allows us to test various gene constructs simultaneously and to acquire a rough idea about the functions of introduced genes in specific cellular environments during development. This method should also be particularly valuable when applied to wild animals such as lampreys, for which the establishment of transgenic lines is inherently difficult due to their complex life cycle and the difficulty of breeding them in the laboratory.

In other studies, this type of technology has been used for misexpression experiments. In ascidians, the transient expression of introduced developmental regulatory genes was followed by large-scale subtractive screens for downstream targets of the regulatory cascade (Takahashi et al., '99). Successful gene transfer into lamprey embryos may provide us with a strategy to directly analyze the function of lamprey developmental genes, and other members of the cascade in which those genes are involved.

The pattern of lamprey embryogenesis is significantly different from that of teleosts such as medaka and zebrafish. Teleost embryos go through discoidal cleavage, and the embryonic part and extraembryonic material such as the yolk are physically separated. When DNA molecules are injected into the egg cytoplasm, they are quickly distributed to multiple cell lineages. Lamprey eggs, on the other hand, are evenly filled with relatively large yolk granules and are opaque like amphibian eggs. Cleavage proceeds holoblastically. These characteristics could potentially cause interference, especially in diffusion of the DNA construct throughout the egg cytoplasm. As the cleavage proceeds, injected DNA may have reached only limited number of blastomeres, which may cause the mosaic expression of reporter genes. Using this method, however, as many as 200 fertilized eggs can be injected during the onecell stage, which should give rise to a considerable number of embryos with exogenous DNA incorporated into their genomes at relatively early stages. Indeed, we obtained multiple embryos injected with OlMA1-GFP or OlMA2-GFP that exhibited distinctive GFP expression in large population of striated muscle cells. By careful observation to ascertain a composite expression pattern from multiple embryos throughout development, the function of the upstream regions that driven the reporter gene expression can be precisely assessed.

\section{Cross-species gene transfer as a tool in the study of evolutionary mechanisms in gene regulation}

Comparisons of the 5, upstream sequences of homologous genes have been made for several developmental genes from various species (for example, Gajewski and Voolstra, 2002). The functional properties of the regulatory regions have also been compared in some transgenic experiments. The activity of cis-regulatory regions of lamprey (Carr et al., '98) and amphioxus (Manzanares et al., 2000) Hox genes have been analyzed in transgenic mice, suggesting the conservation of neuronal enhancers across vertebrate species. The upstream region of another developmental transcription factor, Xenopus Dlx, was tested in transgenic mice and shown to be activated in hair follicles and mammary glands, organs absent in Xenopus (Morasso et al., '95). In the examples described above, however, investigators did not test whether the reporter construct would reproduce the expression pattern of the endogenous gene in the animals from which the upstream sequences originated. The reciprocal transgenic analyses, transferring mouse genes to other animals, have not been conducted. Such analyses are required for any speculation on the conservation and divergence of gene regulatory regions.

In this study, we introduced GFP reporter constructs containing medaka regulatory regions into fertilized lamprey eggs. In the lamprey, both OlMA1- and OlMA2-GFP were expressed in the skeletal 
muscle and only OIMA2-GFP was expressed in cardiac muscle, in accordance with the previously observed expression patterns in medaka (Kusakabe et al., '99). This indicates that lamprey skeletal and cardiac muscle cells contain different sets of transcription factors that can specifically bind to the regulatory regions of medaka muscle actin genes. The regulated activation of medaka muscle actin promoters in lamprey embryos suggests that the skeletal/cardiac musclespecific regulatory mechanisms in extant gnathostomes may have been established before the agnathan/gnathostome divergence.

Characterization of the upstream regions of the lamprey muscle actin genes will add further insight into this picture.

\section{Development of lamprey musculature and evolutionary speculation}

The spaciotemporal expression pattern of OlMA2-GFP in lamprey embryos can be regarded as a marker for muscle-specific regulatory activity, since a lamprey muscle actin gene is expressed at tissues and timing corresponding to that of OlMA2-GFP in the lamprey (R. Kusakabe, unpublished data). In OlMA2-GFP-injected embryos, GFP expression driven by the muscle actin promoter appeared early in somitogenesis, and then spread to head mesoderm derivatives in the pharyngeal arches and head region later in ammocoete larvae (Fig. 3 and 4). Remarkably, however, the hypobranchial muscle, a somitic derivative that migrates around the caudal aspect of the branchial chamber, starts to express GFP in fully grown ammocoete larvae (stage 29; Fig 3G), even later than the initial expression in branchial muscles. Similarly, AchE activity was not detected in the hypobranchial muscle in stage-28.5 larvae (Kuratani et al., '99). It is also noteworthy that none of the OlMA2-GFP-injected embryos expressed GFP in the muscles of the oral region or the velar muscles, both of which are derived from the head mesoderm. In summary, development of the epaxial, hypaxial, branchial, and other head muscles seems to depend on distinct regulatory mechanisms that turn on muscle-specific genes at different times.

The existence of such differential regulatory cascades in skeletal muscle subsets has been reported in mice (Carvajal et al., 2001) and chickens (Hacker and Guthrie, '98; Noden et al., '99; Mootoosamy and Dietrich, 2002). In these animals, genes encoding myogenic regulatory factors (MRFs) Myf5 and Mrf4 were found closely linked on the genome (Carvajal et al., 2001). In mice, the 140-kb upstream sequence of the Myf5 coding region contains multiple regulatory elements that differentially control both Myf5 and Mrf4, in distinct population of skeletal muscle cells (Carvajal et al., 2001; Hadchouel et al., 2000). Noden et al. ('99) showed in chickens that the onset of expression of MRFs is fine-tuned in different musculatures, as it is for downstream muscle structural genes.

We expect that the evolutionary origin of differential control mechanisms of skeletal musculature might be traced back in the study of lamprey, a primitive vertebrate in which some intriguing characteristics are retained in the development of both somitic and head muscles (reviewed in Kuratani et al., 2002). One of the most prominent features is that, as we confirmed in this work (Figs. 3 and 4), some of the most rostral postotic myotomes and the hypobranchial muscle grow rostrally and cover the cranial region; this does not occur in gnathostomes (Kuratani et al., '97, '99, 2002). It is interesting to note that these lamprey-specific myotomes also appear to regulate muscle-related genes as in more posterior trunk myotomes. In gnathostomes, the hypobranchial myoblasts migrates rostroventrally to penetrate the oral floor and gives rise to the tongue muscle (summarized in Dietrich et al., 1999).

Lampreys do not possess a real tongue, and the structure apparently similar to the tongue in lamprey is a head mesoderm derivative (Kuratani et al., '99, 2002). Such differences might be explained by the diversity of structure and regulation of muscle-specific genes encoding proteins involved in the interactions between myogenic cells and surrounding tissues, MRFs, muscle structural proteins including actins. However, the entire structure of the actin multigene family, which in vertebrates consists of multiple striated and smooth muscle-specific genes (Vandekerckhove and Weber, '79), has not been clarified in lamprey. Moreover, no MRF member has been characterized in lamprey. By comparing the detailed expression patterns of these musclespecific genes between agnathans and gnathostomes, it should be possible to characterize the evolutionary pathway of complex head and somitic muscles in extant vertebrates. 


\section{ACKNOWLEDGMENTS}

We thank Dr. Takehiro Kusakabe for technical comments and critical reading of the manuscript, and members of the Ebetsu Fishermen's Association for advice on handling lamprey in the laboratory.

\section{LITERATURE CITED}

Carr JL, Shashikant CS, Bailey WJ, Ruddle FH. 1998. Molecular evolution of Hox gene regulation: cloning and transgenic analysis of the lamprey HoxQ8 gene. J Exp Zool 280:73-85.

Carvajal JJ, Cox D, Summerbell D, Rigby PWJ. 2001. A BAC transgenic analysis of the Mrf4/Myf5 locus reveals interdigitated elements that control activation and maintenance of gene expression during muscle development. Development 128:1857-1868. Cohn MJ. 2002. Lamprey Hox genes and the origin of jaws. Nature 416: 386-387.

Davidson EH. 1999. A view from the genome: spatial control of transcription in sea urchin development. Curr Opin Genet Dev 9:530-541.

Dietrich S, Abou-Rebyeh F, Brohmann H, Bladt F, Sonnenberg-Riethmacher E, Yamaai T, Lumsden A, Brand-Saberi B, Birchmeier C. 1999. The role of SF/HGF and c-Met in the development of skeletal muscle. Development 126: 1621-1629.

Di Gregorio A, Levine M. 2002. Analyzing gene regulation in ascidian embryos: new tools for new perspectives. Differentiation 70:132-139.

Gajewski M, Voolstra C. 2002. Comparative analysis of somitogenesis related genes of the hairy/Enhancer of split class in Fugu and zebrafish. BMC Genomics 3: 21.

Hacker A, Guthrie S. 1998. A distinct developmental programme for the cranial paraxial mesoderm in the chick embryo. Development 125:3461-3472.

Hadchouel J, Tajbakhsh S, Primig M, Chang TH-T, Daubas P, Rocancourt D, Buckingham M. 2000. Modular long-range regulation of Myf5 reveals unexpected heterogeneity between skeletal muscles in the mouse embryo. Development 127: 4455-4467.

Harafuji N, Keys DN, Levine M. 2002. Genome-wide identification of tissue-specific enhancers in the Ciona tadpole. Proc Natl Acad Sci USA 99:6802-6805.

Hardisty MW, Rovainen CM. 1972. Morphological and functional aspects of the muscular system. In: Hardisty MW, Potter I, editors. The biology of lampreys. NY: Academic Press. p 138-231.

Higashijima S, Okamoto H, Ueno N, Hotta Y, Eguchi G. 1997. High-frequency generation of transgenic zebrafish which reliably express GFP in whole muscles or the whole body by using promoters of zebrafish origin. Dev Biol 192:289-299.

Janvier P. 1996. Early Vertebrates. New York: Oxford Scientific Publications.

Jesuthasan S, Subburaju S. 2002. Gene transfer into zebrafish by sperm nuclear transplantation. Dev Biol 242:88-95.

Kroll KL, Amaya E. 1996. Transgenic Xenopus embryos from sperm nuclear transplantations reveal FGF signaling requirements during gastrulation. Development 122: 3173-3183.

Krøvel AV, Olsen LC. 2002. Expression of a vas::EGFP transgene in primordial germ cells of the zebrafish. Mech Dev 116:141-150.

Kuraku S, Hoshiyama D, Katoh K, Suga H, Miyata T. 1999. Monophyly of lampreys and hagfishes supported by nuclear DNA-coded genes. J Mol Evol 49:729-735.

Kuratani S, Ueki T, Aizawa S, and Hirano, S. 1997. Peripheral development of cranial nerves in a cyclostome, Lampetra japonica: morphological distribution of nerve branches and the vertebrate body plan. J Comp Neurol 384:483-500.

Kuratani S, Horigome N, Hirano S. 1999. Developmental morphology of the head mesoderm and reevaluation of segmental theories of the vertebrate head: evidence from embryos of an agnathan vertebrate, Lampetra japonica. Dev Biol 210:381-400.

Kuratani S, Kuraku S, Murakami Y. 2002. Lamprey as an evodevo model: lessons from comparative embryology and molecular phylogenetics. Genesis 34:175-183.

Kuratani S, Nobusada Y, Horigome N, Shigetani Y. 2001. Embryology of the lamprey and evolution of the vertebrate jaw: insights from molecular and developmental perspective. Phil Trans R Soc Lond B 356:1615-1632.

Kusakabe T. 1997. Ascidian actin genes: Developmental regulation of gene expression and molecular evolution. Zool Sci 14:707-718.

Kusakabe T, Suzuki N. 2001. A cis-regulatory element essential for photoreceptor cell-specific expression of a medaka retinal guanylyl cyclase gene. Dev Genes Evol 211: 145-149.

Kusakabe R, Kusakabe T, Suzuki N. 1999. In vivo analysis of two striated muscle actin promoters reveals combinations of multiple regulatory modules required for skeletal and cardiac muscle-specific gene expression. Int J Dev Biol 43:541-554. Mallatt J, Sullivan J. 1998. 28S and 18S rDNA sequences support the monophyly of lampreys and hagfishes. Mol Biol Evol 15:1706-1718.

Manzanares M, Wada H, Itasaki N, Trainor PA, Krumlauf R, Holland PWH. 2000. Conservation and elaboration of Hox gene regulation during evolution of the vertebrate head. Nature 408:854-857.

Mello C, Fire A. 1995. DNA transformation. In: Epstein HF, Shakes DC, editors. Caenorhabiditis elegans: Modern Biological Analysis of an Organism. New York: Academic Press. p 451-482. 
Mootoosamy RC, Dietrich S. 2002. Distinct regulatory cascades for head and trunk myogenesis. Development 129:573-583.

Morasso MI, Mahon KA, Sargent TD. 1995. A Xenopus Distal-less gene in transgenic mice: conserved regulation in distal limb epidermis and other sites of epithelialmesenchymal interaction. Proc Natl Acad Sci USA 92: 3968-3972.

Mu"ller U. 1999. Ten years of gene targeting: targeted mouse mutants, from vector design to phenotype analysis. Mech Dev $82: 3-21$.

Murakami Y, Ogasawara M, Sugahara F, Hirano S, Satoh N, Kuratani S. 2001. Identification and expression of the lamprey Pax6 gene: evolutionary origin of the segmented brain of vertebrates. Development 128:3521-3531.

Myojin M, Ueki T, Sugahara F, Murakami Y, Shigetani Y, Aizawa S, Hirano S, Kuratani S. 2001. Isolation of Dlx and Emx cognates in an agnathan species, Lampetra japonica, and their expression patterns during embryonic and larval development: Conserved and diversified regulatory patterns of homeobox genes in vertebrate head evolution. J Exp Zool (Mol Dev Evol) 291:68-84.

Noden DM, Marcucio R, Borycki A-G, Emerson CP Jr. 1999. Differentiation of avian craniofacial muscles: I. Patterns of regulatory gene expression and myosin heavy chain synthesis. Dev Dyn 216:96-112.

Ogasawara M, Shigetani Y, Hirano S, Satoh N, Kuratani S. 2000. Pax1/Pax9-related genes in an agnathan vertebrate, Lampetra japonica: expression pattern of LjPax9 implies sequential evolutionary events toward the gnathostome body plan. Dev Biol 223:399-410.

Rong YS, Titen SW, Xie HB, Golic MM, Bastiani M, Bandyopadhyay P, Olivera BM, Brodsky M, Rubin GM, Golic KG. 2002. Targeted mutagenesis by homologous recombination in D. melanogaster. Genes Dev 16:1568- 1581 .

Shigetani Y, Sugahara F, Kawakami Y, Murakami Y, Hirano S, Kuratani, S. 2002. Heterotopic shift of epithelial mesenchymal interactions in vertebrate jaw evolution. Science 296:1316-1319.

Steinberg M. 1957. A nonnutrient culture medium for amphibian embryonic tissues. Carnegie Institution of Washington Year Book 56:347-348.

Swartz M, Eberhart J, Mastick GS, Krull CE. 2001. Sparking new frontiers: using in vivo electroporation for genetic manipulations. Dev Biol 233:13-21.

Tahara Y. 1988. Normal stages of development in the lamprey, Lampetra reissneri (Dybowski). Zool Sci 5:109-118. Takahashi H, Hotta K, Erives A, Di Gregorio A, Zeller RW, Levine M, Satoh, N. 1999. Brachyury downstream notochord differentiation in the ascidian embryo. Genes Dev 13: 1519-1523.

Vandekerckhove J, Weber K. 1979. The complete amino acid sequence of actins from bovine aorta, bovine heart, bovine fast skeletal muscle, and rabbit slow skeletal muscle. Differentiation 14:123-133. 
A
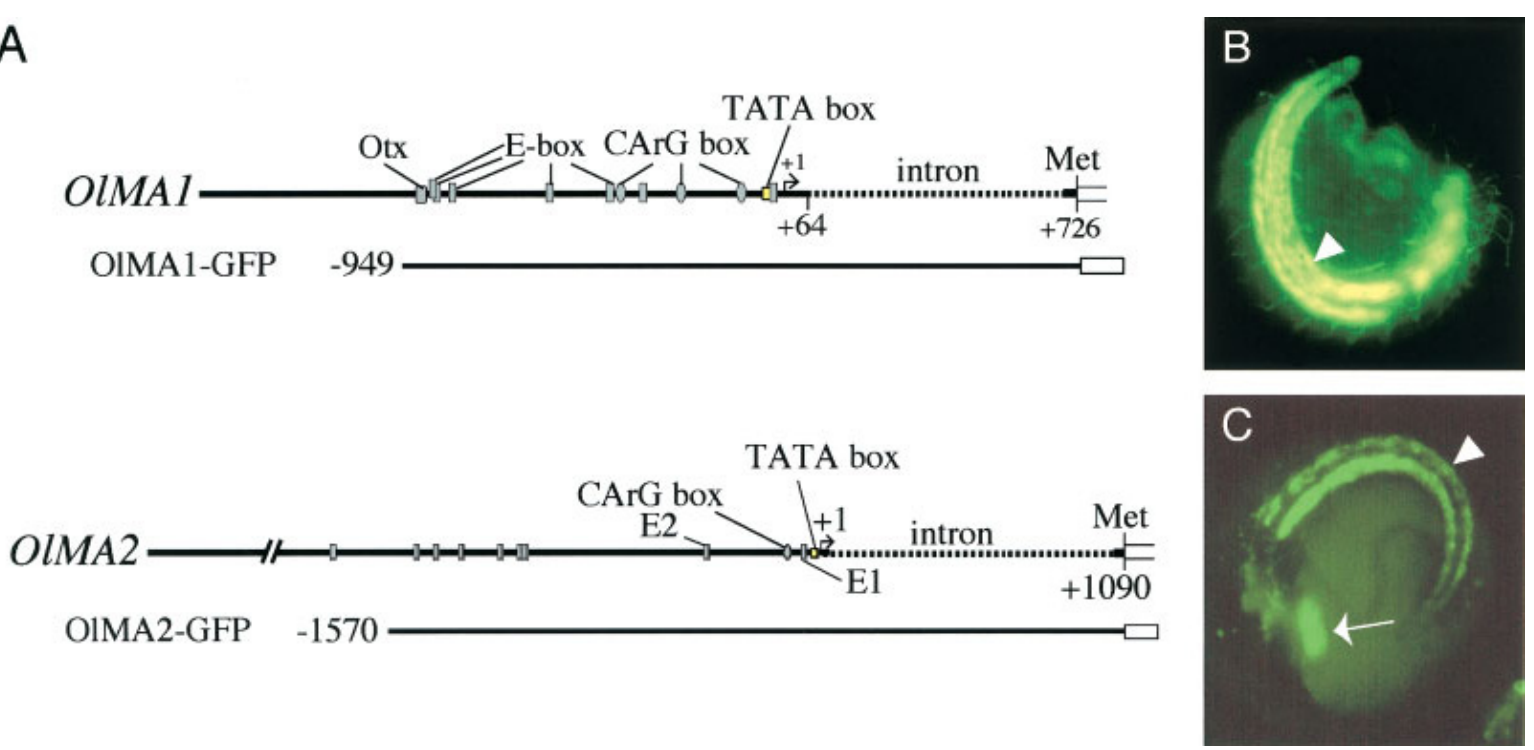

Fig. 1. Structures and expression of medaka muscle actin- GFP constructs used in this study. A. The genomic structures of endogenous medaka actin genes (top) and corresponding fusion gene constructs (Kusakabe et al., '99). Broken lines correspond to the first intron of the gene. "Met" indicates the start Met codon of actin open reading frames (ORF). In all the fusion genes, the actin ORF was replaced with the GFP ORF, as indicated by a white box. B. A five-day medaka embryo injected with an OlMA1-GFP fusion construct. Strong signal is seen in somitic muscle (white arrowhead). C. A four-day medaka embryo injected with OIMA2-GFP fusion construct. GFP expression is strong in both somitic (white arrowhead) and cardiac (white arrow) muscles. 

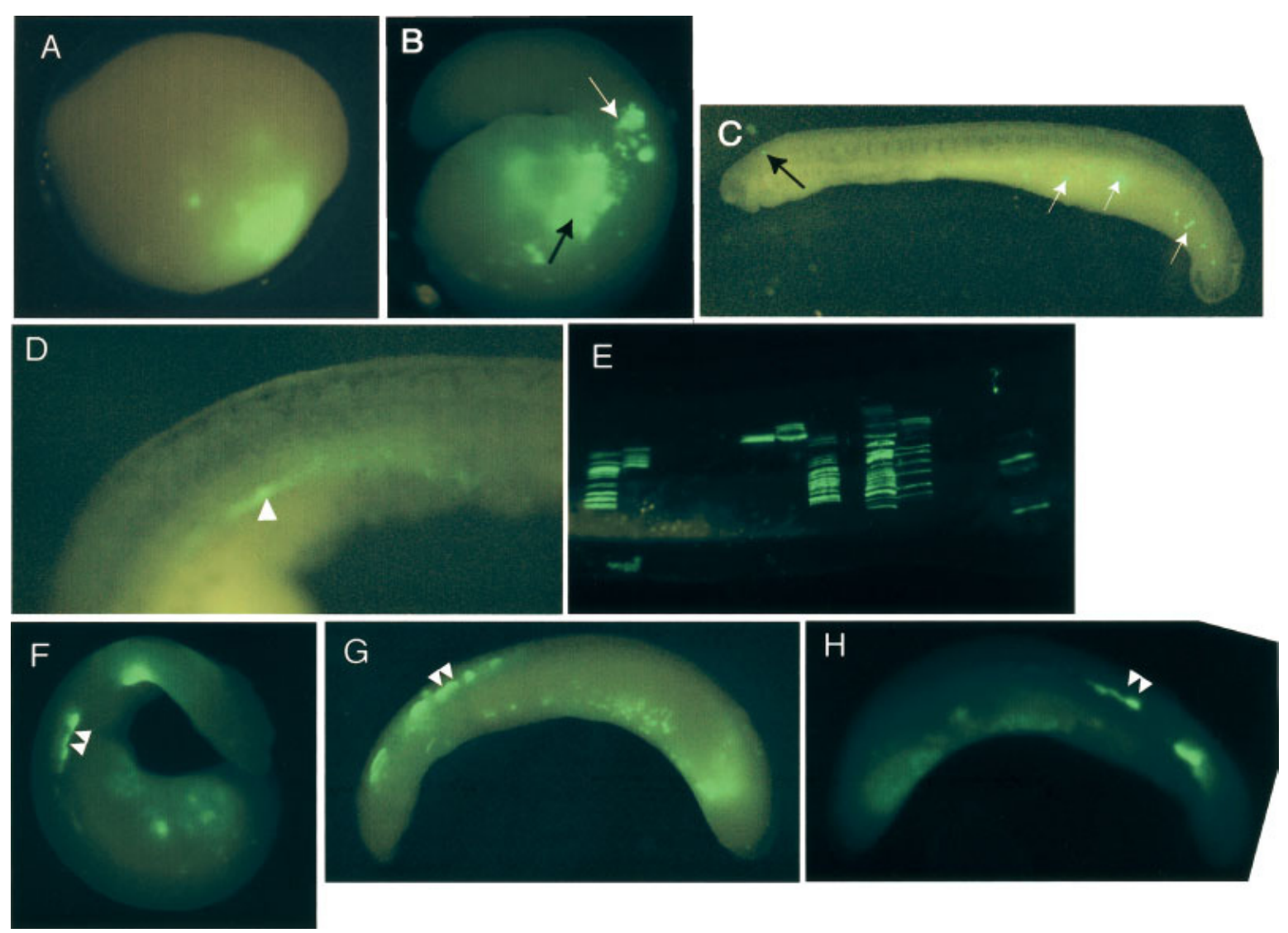

Fig. 2. Expression of pEGFP-N1 or OlMA1-GFP in lamprey embryos. A. A stage-18 embryo injected with pEGFP-N1. Anterior is to the right. GFP-positive yolk or epidermal cells form a cluster in the ventral trunk area. B. A stage-22 embryo injected with pEGFP-N1. Strong signal is evident in the ventrolateral trunk (black arrow) and epidermal cells right above the forming somites (white arrow). C. Another example of pEGFP-N1-injected embryos at stage 26. In addition to patchy, scattered signal seen in the ventrolateral trunk area (white arrows), a single cell with signal is visible in the head (black arrow). D. A pEGFP-N1-injected embryo at stage 27. Anterior is to the right. Putative pronephric duct has strong GFP signal (white arrowhead). E. Another pEGFP-N1-injected embryo at the ammocoete larval stage. Anterior is to the right. The body-wall muscle cells are partly GFP-positive. F. A stage 24 embryo injected with OlMA1-GFP. Somite-specific signal (double arrowhead) is evident. G and $\mathrm{H}$. Stage 25 of the same embryo shown in F, in left $(\mathrm{G})$ and right $(\mathrm{H})$ lateral views. Expression patterns of somite-specific signal (double arrowhead) are different on the two sides. 

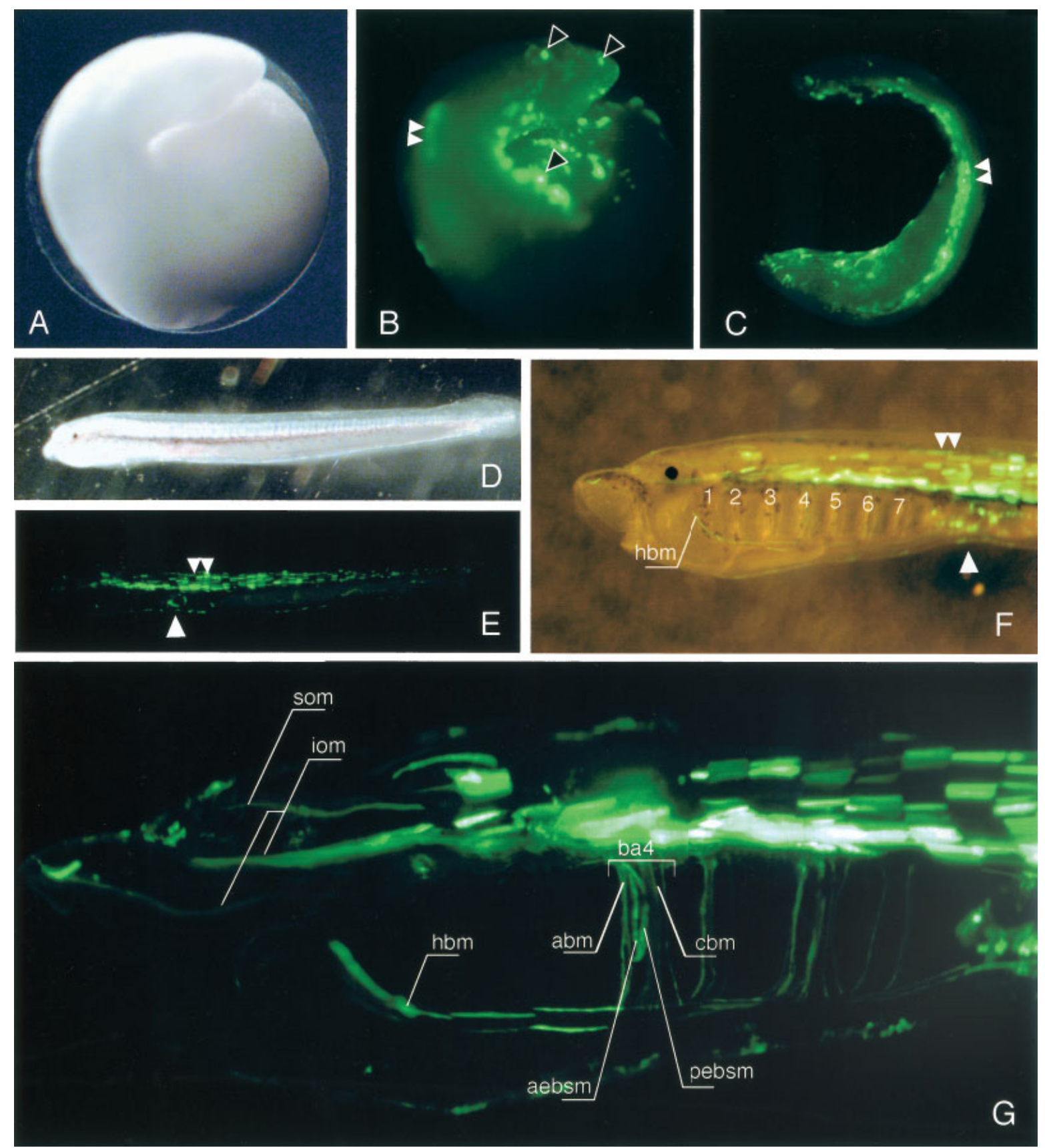

Fig. 3. Expression sequence of OlMA2-GFP in lamprey embryo \#1 during development. A. Stage-23 embryo, photographed in bright field. B. The same view as in A, observed under an excitation light. Patchy signals (black arrowhead) are seen in the head and ventral trunk. Initial muscle-specific expression (double white arrowhead) was detected in the dorsolateral trunk. C. At stage 25, muscle-specific signal is evident (white arrow). Putative non-specific signal is seen in the epidermis. D. On day 24, ammocoete larval stage. E. The same view as in D, under an excitation light. Signal occurs in both somitic (double white arrowhead) and cardiac (white arrowhead) muscle. F. The head of the day-36 larva. Numbers indicate the positions of seven branchial arches. The signal is strong in somitic (double white arrowhead) and cardiac (white arrowhead) muscle. Branchial arches 3-7 and hypobranchial muscle (hbm) express GFP signal in muscle fibers. G. Higher magnification of F. Abbreviations: abm, adductor branchial muscle; aebsm, anterior external branchial sphincter muscle; ba4, branchial arch 4; cbm, constrictor branchial muscle; iom, infraoptic muscle; pebsm, posterior external branchial sphincter muscle; som, supraoptic muscle. 

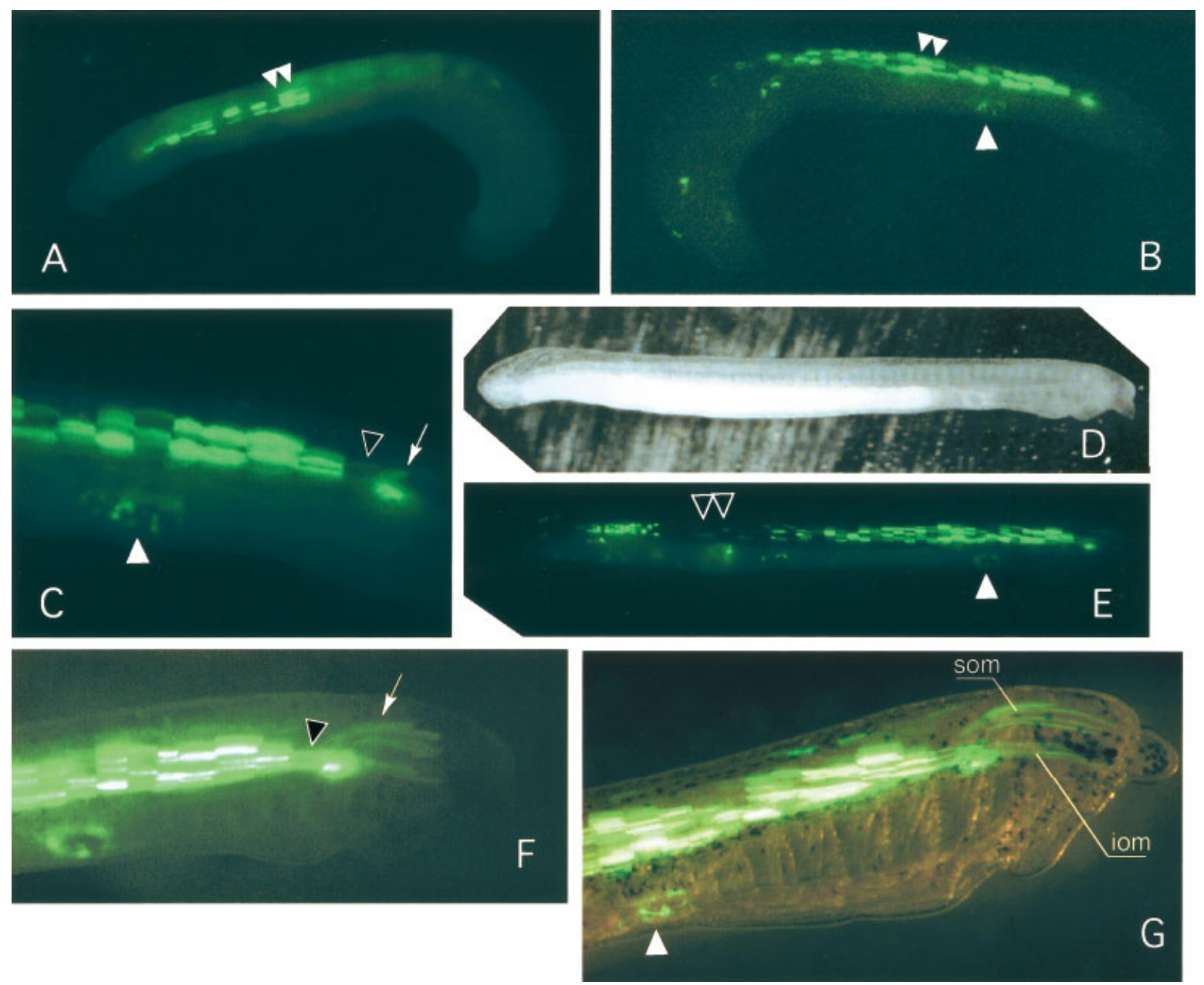

Fig. 4. Expression sequence of OlMA2-GFP in lamprey embryo \#7. A. Stage 25. Viewed from the left side. Anterior is to the left. Somites are marked with strong GFP signal (double white arrowhead). B-G. Anterior is to the right. B. The right side of the same embryos as shown in A. There are more somites expressing signal (double white arrowhead) than are seen in the view from the left side. Pulsating cardiac muscle (white arrowhead) expressing strong signal is also visible. C. Higher magnification of the head part from B. Signal is absent from somite 3 (black arrowhead). Somite 2 splits into dorsal and ventral halves (white arrow). D. Day-18 ammocoete larva in bright field. E. The same view as in D, observed under an excitation light. Strong signal in somitic muscle is absent only from the mid-part of the trunk (double black arrowhead). The heart is out of focus (white arrowhead). F. Higher magnification of the head from E. Muscle fibers from somite 2 extend anteriorly (white arrow). G. Head part of day-24 ammocoete larva. Cardiac muscle (white arrowhead), somitic muscle, and head muscle derived from somite 2 ("som" and “'iom,'” Kuratani et al., '99) show strong signal. Abbreviations: iom, infraoptic muscle; som, supraoptic muscle. 